\title{
Social egg freezing: risk, benefits and other considerations
}

\author{
Angel Petropanagos PhD, Alana Cattapan MA, Françoise Baylis PhD, Arthur Leader MD
}

CMAJ Podcasts: author interview at soundcloud.com/cmajpodcasts/egg-freezing

Competing interests: Arthur Leader is a partner at the Ottawa Fertility Centre. No other competing interests were declared.

\section{Correspondence to:} Angel Petropanagos, angel.p@dal.ca

CMAJ 2015. DOI:10.1503 /cmaj.141605
I n late 2012, the American Society for Reproductive Medicine (ASRM) and the Society for Assisted Reproductive Technology (SART) announced that they would no longer consider oocyte cryopreservation (i.e., egg freezing) to be experimental. In their joint practice guideline, published in early 2013, ${ }^{1}$ the ASRM and SART specifically cautioned against the use of egg freezing as a guard against age-related fertility decline, owing to limited data about the safety, efficacy, cost-effectiveness and emotional risks of egg freezing for healthy women of reproductive age. In 2014, the American College of Obstetricians and Gynecologists (ACOG) endorsed the ASRM-SART guideline. Notwithstanding these cautions, the use of social egg freezing (i.e., nonmedical egg freezing) is increasing in the United States. This increase has been linked to a number of complex and interrelated reasons for delayed childbearing, including personal, professional, financial and psychological factors. ${ }^{2}$

In October 2014, the Canadian Fertility and Andrology Society released its position statement on egg freezing, describing it as "an option for women wishing to preserve their fertility in the face of anticipated decline." ${ }^{3}$ No new clinical data were presented in support of this position statement - a statement that directly contradicts both the ASRM-SART practice guideline and the ACOG policy.

Recent media attention has contributed to the increasing public awareness of egg freezing in Canada. Consider, for example, media interest in announcements by Facebook and Apple that they would provide limited insurance coverage for the cost of egg freezing as an employee benefit. ${ }^{4}$ With growing public awareness of social egg freezing, Canadian women may increasingly approach physicians in search of information and advice about the procedure. Family physicians are uniquely positioned at the front lines of medical care to provide information to women who ask about egg freezing. This paper outlines medical, financial and societal implications of social egg freezing that are relevant in providing accurate and balanced information for such discussions.

\section{How are human oocytes retrieved and frozen?}

Egg freezing is a process that involves the hormonal stimulation of the ovaries, followed by transvaginal retrieval and subsequent freezing and storage of a woman's viable eggs. ${ }^{1}$ Although egg freezing can occur using slow-freezing or vitrification (flash-freezing) protocols, the latter technology has increased oocyte survival post-thaw and improved pregnancy rates, ${ }^{1,5}$ and is the method recommended by the relevant professional medical associations. Vitrification involves the use of cryoprotectants and ultrarapid cooling in an effort to solidify the cell to avoid the formation of ice crystals. The ASRM-SART practice guideline estimates that the survival rate of oocytes after vitrification and thawing is $90 \%-97 \%$, the fertilization rate is $71 \%-79 \%$ and the implantation rate is $17 \%-41 \%$. The clinical pregnancy rate per vitrified and thawed oocyte is $4.5 \%-12 \%{ }^{1}$ However, it should be noted that these data are generally derived from oocytes obtained from women less than 30 years of age. Clinical pregnancy rates decline with advanced maternal age at the time of freezing. ${ }^{1}$ Furthermore, the ASRM estimates that the live birth rate is $2 \%-12 \%$ for women under 38 years of age. ${ }^{6}$ Studies examining the success rates 
of social egg freezing remain limited, and egg freezing does not guarantee a successful pregnancy or live birth.

\section{What are the potential benefits of social egg freezing and in vitro fertilization?}

Egg freezing has been used since the late 1990s for fertility preservation for young women with cancer who are at risk of sterility as a result of their cancer or its treatment. ${ }^{7,8}$ Social egg freezing is typically offered to women under 38 years of age who want to preserve the option of having healthy, genetically related children at a later date. ${ }^{9}$

Social egg freezing, followed by in vitro fertilization (IVF) and embryo transfer, offers two important benefits to women who anticipate becoming pregnant at an advanced age: it provides them with the possibility of becoming a genetic parent using their frozen-thawed eggs, and it reduces the risk of having children with chromosomal abnormalities associated with ovarian aneuploidy..$^{10}$ In addition, for women who do not have a partner or for women who have moral concerns about the status of a developing embryo, egg freezing may be a preferable alternative to embryo freezing.

\section{What are the medical risks of social egg freezing and IVF?}

Medical risks related to ovarian stimulation and egg retrieval should be communicated to all women who ask about social egg freezing. Young, healthy, fertile women should know that the data on the effects of ovarian stimulation and egg retrieval are limited insofar as they are based on the experiences in a different patient population (i.e., older women with subfertility or infertility).

Perhaps the most important medical risks associated with egg freezing are those that can result from ovarian stimulation, such as ovarian hyperstimulation syndrome. Mild-to-moderate ovarian hyperstimulation syndrome involves fatigue, nausea, headaches, abdominal pain, breast tenderness and irritability, ${ }^{11}$ but these adverse effects can usually be well-controlled. However, $0.1 \%-2 \%$ of patients may experience severe ovarian hyperstimulation syndrome, ${ }^{12}$ resulting in blood clots, shortness of breath, abdominal pain, dehydration and vomiting that necessitates admission to hospital. ${ }^{13}$ In rare instances, death can result; ${ }^{13}$ however, no such deaths have been reported in Canada to date.
Suggestions have been made that ovarian stimulation may increase the risk of breast, uterine and other cancers. However, reports of cancer are limited and conflicting; ${ }^{14-16}$ further research is needed.

Women who attempt to achieve a pregnancy using their frozen-thawed eggs will be subject to the risks of IVF. These risks include multiple pregnancy, pregnancy-related high blood pressure, premature delivery, operative delivery and infants with low birth weight. ${ }^{17,18}$

\section{What are the medical risks of pregnancy at an advanced age?}

Medical risks to women and their future offspring that are associated with pregnancy at an advanced age (i.e., expected delivery after age $35 \mathrm{yr}$ ) should be communicated to women who ask about social egg freezing. ${ }^{10}$

\section{Risks to women}

Women who become pregnant at an advanced age are at an increased risk of gestational diabetes, preeclampsia, cesarean delivery and preterm delivery of a baby with low birth weight. However, these risks vary widely, depending on the woman's health status, and increase with maternal age at delivery. ${ }^{10}$

\section{Risks to offspring}

In addition to the harms associated with premature birth and low birth weight, there is evidence of "a slight increase in the risk of congenital structural abnormalities" with IVF, and some research suggests that there may be a small but increased risk of cancer and structural cardiac anomalies for these children. ${ }^{19}$ Further research is needed to confirm the risks to children born of IVF and to determine whether there are risks specifically associated with births from frozen eggs.

\section{What are the financial costs of social egg freezing?}

In Canada, the costs and availability of social egg freezing vary by province and by clinic. Reported costs range between $\$ 5000$ and $\$ 10000$ per stimulated cycle. ${ }^{20}$ These costs may include consultations, laboratory fees, medications, the egg retrieval procedure, freezing and storage, although not all of these costs are always included in clinic estimates. Storage fees (estimated at between $\$ 300$ and $\$ 500$ per year) may add substantial costs to social egg freezing over time; arguably, this expense is only a sound investment if egg freezing 
is followed by IVF and embryo transfer that results in a successful pregnancy and a live birth.

Physicians should inform women that provincial health insurance programs do not currently cover social egg freezing costs and no provinces offer funding for IVF after social egg freezing. Patients should give careful consideration to both present and future costs. Given these high costs, social egg freezing is very likely an option for only a small group of privileged women.

\section{What are the societal implications of social egg freezing?}

Social egg freezing uses medical technology to respond to a nonmedical problem - natural aging. Physicians should therefore move beyond a discussion of the potential benefits, risks and financial costs to address societal implications.

Increased media attention, as well as employer and private insurance coverage of social egg freezing, may result in growing pressures on young women to freeze their eggs. ${ }^{21-23}$ Media coverage often emphasizes the potential benefits of egg freezing and ignores or downplays the associated risks. ${ }^{24}$ With social egg freezing presented as a valuable reproductive option, some women may come to believe that freezing their eggs is the best way (if not the only way) to secure the opportunity of having a healthy, genetically related child in the future.

The portrayal of social egg freezing as a "backup plan" or "fertility insurance" that allows young fertile women to take the time to find suitable long-term partners, complete their education, achieve financial stability or advance their career goals without having to worry about their future fertility may strongly influence the ways in which some women's reproductive choices are shaped. Physicians should be careful not to place additional pressure on women by portraying egg freezing as something that they should choose to avoid future regret.

Pressures for women to freeze their eggs occur within a broader social context that strongly pushes women to become mothers. Egg freezing as a way of preserving women's reproductive options reinforces the social norms and expectations that construe motherhood as a central aspect of womanhood. Women are encouraged to freeze their eggs as a way to "have it all" (that is, to have both a family and a career), implying that for those women who want both these things, egg freezing makes this possible. ${ }^{24}$ Although individual women may benefit from egg freezing to satisfy their reproductive desires, physicians should not assume that having a genetically related child is equally important to all women who ask about social egg freezing.

For women who do want genetically related children and who wish to avoid the difficulties associated with infertility, ${ }^{25}$ the pressure to use egg freezing unduly places reproductive responsibilities on individual women and obscures the influence of social structures that can contribute to delayed childbearing in the first place. For example, in the absence of funded child care programs that can make child-bearing and child-rearing at a younger age more likely and easier, the decision to delay child-bearing may not be a choice at all.

Social egg freezing is inaccessible to women without substantial financial resources, and it is misleading to frame social egg freezing as a benefit to all women. It is important to consider the ways in which this technology may work to privilege the family-making projects of already privileged women, and to exclude others who cannot pay for it.

Social egg freezing reinforces assumptions about the value of having genetically related children, which may not be of equal importance to all women. It is important to outline relative potential benefits and risks of all options for family-making.

\section{Conclusion}

In light of the controversial nature of social egg freezing, with competing perspectives and information available from a variety of sources, family physicians have a unique opportunity to assist women in accessing accurate and balanced information about their reproductive health. This information should be provided to all women who ask about social egg freezing, regardless of sexual orientation, age, disability, health, relationship or socioeconomic status. Family physicians should frame discussions about this practice within the broader context of reproductive health and family-making to assist women in making informed choices.

\section{References}

1. Practice Committees of American Society for Reproductive Medicine, Society for Assisted Reproductive Technology. Mature oocyte cryopreservation: a guideline. Fertil Steril 2013; 99:37-43.

2. Hodes-Wertz B, Druckenmiller S, Smith M, et al. What do reproductive-aged women who undergo oocyte cryopreservation think about the process as a means to preserve fertility? Fertil Steril 2013;100:1343-9.

3. Position statement on egg freezing. Montréal: Canadian Fertility and Andrology Society; 2014. Available: www.cfas.ca/images/ stories/pdf/Position_Statement_Egg_Freezing_2014-10-21.pdf (accessed 2015 Mar. 1).

4. Ortutay B. Facebook, Apple offer to freeze female employees' eggs. Waterloo Region Record 2014 Oct. 12;Sect. C:12.

5. Al-Azawi T, Tavukcuoglu S, Khaki AA, et al. Cryopreservation of human oocytes, zygotes, embryos and blastocysts: a comparison study between slow freezing and ultra rapid (vitrification) methods. Middle East Fertil Soc J 2013;18:223-32. 
6. Fact sheet: Can I freeze my eggs to use later if I'm not sick? Birmingham (AL): American Society for Reproductive Medicine; 2014. Available: www.sart.org/uploadedFiles/ASRM Content/ Resources/Patient_Resources/Fact_Sheets_and_Info_Booklets/ Can_I_freeze_my_eggs_to_use_later_if_Im_not_sick-FINAL_8 -13-14.pdf (accessed 2015 Mar. 1)

7. Porcu E, Notarangelo L, Bazzocchi A, et al. Early and recent history of oocyte cryopreservation in human IVF. In: Borini A, Coticchio, editors. Preservation of human embryos: from cryobiology science to clinical applications. London (UK): Informa Healthcare; 2009.

8. Porcu E, Ciotti P, Venturoli S, editors. Handbook of human oocyte cryopreservation. Cambridge: Cambridge University Press; 2013.

9. Boldt J. Current results with slow freezing and vitrification of the human oocyte. Reprod Biomed Online 2011;23:314-22

10. Liu K, Case A. Advanced reproductive age and fertility. J Obstet Gynaecol Can 2011;33:1165-75.

11. Suthersan D, Kennedy S, Chapman M. Physical symptoms throughout IVF cycles. Hum Fertil (Camb) 2011;14:122-8.

12. Joint Society of Obstetricians and Gynaecologists of Canada and Canadian Fertility and Andrology Society. The diagnosis and management of ovarian hypestimulation syndrome. $J$ Obstet Gynaecol Can 2011;268:1156-62.

13. Fact sheet: Ovarian hyperstimulation syndrome (OHSS). Birmingham (AL): American Society for Reproductive Medicine; 2014. Available: www.asrm.org/uploadedFiles/ASRM_Content/ Resources/Patient_Resources/Fact_Sheets_and_Info_Booklets/ OHSS_final_1-5-12.pdf (accessed 2015 Mar. 1).

14. Calderon-Margalit R, Friedlander Y, Yanetz R, et al. Cancer risk after exposure to treatments for ovulation induction. Am J Epidemiol 2009;169:365-75.

15. Rizzuto I, Behrens RF, Smith LA. Risk of ovarian cancer in women treated with ovarian stimulating drugs for infertility. Cochrane Database Syst Rev 2013;(8):CD008215.

16. Ovarian simulation for IVF does not increase risk of endometrial or colorectal cancer: results from a nationwide cohort study [press release]. 2014 Oct. 21. Washington (DC): American Society for Reproductive Medicine Office of Public Affairs. Available: www.asrm.org/Ovarian_Stimulation_for_IVF_Does_Not_Increase _Risk_of_Endometrial_or_Colorectal_Cancer_Results_from_a_ Nationwide_Cohort_Study/ (accessed 2015 Mar. 15).

17. Risks of in vitro fertilization (IVF). Birmingham (AL): American Society for Reproductive Medicine; 2014. Available: www.asrm
.org/uploadedFiles/ASRM_Content/Resources/Patient_Resources /Fact_Sheets_and_Info_Booklets/risksofivf.pdf. (accessed 2015 Mar. 1).

18. Gelbaya TA. Short- and long-term risks to women who conceive through in vitro fertilization. Hum Fertil (Camb) 2010; 13:19-27.

19. Okun N, Sierra S. SOGC clinical practice guidelines: pregnancy outcomes after assisted human reproduction. $J$ Obstet Gynaecol Can 2014;36:64-83.

20. Shupac J. Motherhood postponed: freeze now, hatch later. Canadian Business 2013 Jan.16. Available: www.canadianbusiness. com/economy/motherhood-postponed/ (accessed 2014 Dec. 12)

21. Baylis F. Left out in the cold: arguments against non-medical oocyte cryopreservation. J Obstet Gynaecol Can 2015;37:64-67.

22. Kheiriddin T. The newest job perk. National Post 2014 Oct. 16; Sect A:8.

23. Ravitsky V, Lemoine M-E. We need a culture thaw, not frozen eggs. The Globe and Mail 2014 Oct. 18; Sect F:2.

24. Cattapan A, Hammond K, Haw J, et al. Breaking the ice: young feminist scholars of reproductive politics reflect on egg freezing. IJFAB 2014;7:236-47.

25. Caron SL, Ferland P. Exploring the long-term impact of female infertility: a qualitative analysis of interviews with postmenopausal women who remained childless. Fam J (Alex Va) 2013;21:180-8.

Affiliations: Novel Tech Ethics (Petropanagos, Cattapan, Baylis), Faculty of Medicine, Dalhousie University, Halifax, NS; Department of Obstetrics and Gynecology, University of Ottawa, and Ottawa Fertility Centre (Leader), Ottawa, Ont.

Funding: Research for this paper was funded by Canadian Institutes of Health Research grant no. EOG111389, "A comparative study of assisted human reproduction patients' views about the donation of eggs and embryos for scientific and clinical research."

Contributors: Angel Petropanagos and Alana Cattapan contributed to the conception, drafting, analysis and revisions of this work. Françoise Baylis contributed to the drafting, analysis and revisions. Arthur Leader contributed to the analysis and revisions. All authors approved the final version to be published and agree to act as guarantors of the work. 